\title{
Digital Watermarking of Spectral Images with Three-Dimensional Wavelet Transform
}

\author{
Arto Kaarna ${ }^{1}$ and Jussi Parkkinen ${ }^{2}$ \\ 1 Lappeenranta University of Technology \\ Department of Information Technology \\ P.O. Box 20, FIN-53851, Lappeenranta, Finland \\ 2 University of Joensuu \\ Department of Computer Science \\ P.O. Box 111, FIN-80101, Joensuu, Finland \\ Arto.Kaarna@lut.fi, Jussi.Parkkinen@joensuu.fi
}

\begin{abstract}
We propose a watermarking method for spectral images. The watermark is embedded in the three-dimensional wavelet domain of the image. Before embedding, the gray-scale watermark is transformed with the two-dimensional wavelet transform. After embedding the inverse three-dimensional wavelet transform reconstructs the image now containing the watermark. The strength of embedding is controlled by perceptual constraints and reconstruction quality. The experiments indicate, that the robustness against attacks like PCA/wavelet compression is good.
\end{abstract}

\section{Introduction}

Copyright protection is becoming more important in open networks since digital copying maintains the original data and copying can be done easily and at high speed. Digital watermarks offer a possibility to protect copyrighted data in the information society. The watermarking procedure should maintain the following properties: the watermark should be undetectable and secret for an unauthorized user, the watermark should be invisible or inaudible in the information carrying signal and finally, the watermark should be robust on the possible attacks [1], [2], [3], [4].

Several watermarking techniques have been developed for gray-scale and RGBcolor images [5]. Most often the watermark is embedded in the transform domain of the image [6], [7], but also the original spatial domain has been used [8]. For RGB-color images different color spaces are considered for watermarking [9].

Spectral images are often used in remote sensing and nowadays, their utilization has expanded to industrial applications for example in quality control. Spectral images constitute a new area for watermarking technology. The transform domain has been popular also in spectral image watermarking. One solution for spectral images is to embed a watermark in the transform domain of each band. The transform domain is calculated with the discrete cosine transform or the wavelet transform [10] or with the Hadamard transform [11]. The watermarks in the above references are binary or grayscale images. 
In this study we embed the gray-scale watermark into a spectral image in the transform domain of the three-dimensional wavelet transform. The report is organized as follows: the three-dimensional wavelet transform is described in Section 2. The watermarking procedure is established in Section 3, attacks are described in section 4, experiments with the procedure are in Section 5 and the conclusions are drawn in Section 6.

\section{Three-Dimensional Wavelet Transform}

In the multiresolution analysis [12,13] a function $f \in L^{2}(R)$ is approximated with successive subspaces $\ldots V_{j} \subset V_{j-1} \ldots \subset L^{2}(R)$. The scaling function $\left\{\phi_{j, n}\right\}_{n \in Z}$ is an orthonormal basis for $V_{j}$ for all $j \in Z$. $W_{j}$ is an orthogonal complement of $V_{j}$ in $V_{j-1}$, $V_{j-1}=V_{j} \oplus W_{j}, \quad W_{j} \perp V_{j}$ and consequently, for $j<J V_{j}=V_{J} \bigoplus_{k=0}^{J-j-1} W_{J-k}$.

With multidimensional signals the similar multiresolution condition holds. A theorem says [13], that the family obtained by dilating and translating the $2^{p}-1$ wavelets for $\alpha \neq 0$

$$
\left\{2^{-\frac{p j}{2}} \Psi^{\alpha}\left(\frac{x_{1}-2^{j} n_{1}}{2^{j}}, \ldots, \frac{x_{p}-2^{j} n_{p}}{2^{j}}\right)\right\}_{1 \leq \alpha<2^{p},\left(j, n_{1}, \ldots, n_{p}\right) \in Z^{p+1}}
$$

is an orthonormal basis for $L^{2}\left(R^{p}\right)$.

Spectral images belong to the class of multidimensional signals with dimensionality of three. If the spectral domain is considered similar to the spatial domain, then the separable multiresolution for spectral images is

$$
\begin{aligned}
\mathbf{V}_{j-1}= & V_{j-1} \otimes V_{j-1} \otimes V_{j-1}=\left(V_{j} \oplus W_{j}\right) \otimes\left(V_{j} \oplus W_{j}\right) \otimes\left(V_{j} \oplus W_{j}\right) \\
= & \left(V_{j} \oplus W_{j}\right) \otimes\left\{\left(V_{j} \otimes V_{j}\right) \oplus\left(V_{j} \otimes W_{j}\right) \oplus\left(W_{j} \otimes V_{j}\right) \oplus\left(W_{j} \otimes W_{j}\right)\right\} \\
= & \left(V_{j} \otimes V_{j} \otimes V_{j}\right) \oplus\left\{\left(V_{j} \otimes V_{j} \otimes W_{j}\right) \oplus\left(V_{j} \otimes W_{j} \otimes V_{j}\right) \oplus\right. \\
& \left(V_{j} \otimes W_{j} \otimes W_{j}\right) \oplus\left(W_{j} \otimes V_{j} \otimes V_{j}\right) \oplus\left(W_{j} \otimes V_{j} \otimes W_{j}\right) \oplus \\
& \left.\left(W_{j} \otimes W_{j} \otimes V_{j}\right) \oplus\left(W_{j} \otimes W_{j} \otimes W_{j}\right)\right\} \\
= & \mathbf{V}_{j} \oplus \mathbf{W}_{j}
\end{aligned}
$$

The scaling function for the basis $\mathbf{V}_{0}$ is

$$
\boldsymbol{\Phi}_{0, n_{1}, n_{2}, n_{3}}\left(x_{1}, x_{2}, x_{3}\right)=\phi\left(x_{1}-n_{1}\right) \phi\left(x_{2}-n_{2}\right) \phi\left(x_{3}-n_{3}\right), n_{1}, n_{2}, n_{3} \in Z
$$

and now, the filtering of the multispectral image is done using one scaling function $\phi\left(x_{i}\right)$ and seven wavelets $\psi\left(x_{i}\right)$, which are defined as

$$
\begin{array}{ll}
\Phi^{s, a}\left(x_{1}, x_{2}, x_{3}\right)=\phi\left(x_{1}\right) \phi\left(x_{2}\right) \phi\left(x_{3}\right) & \Psi^{h, a}\left(x_{1}, x_{2}, x_{3}\right)=\phi\left(x_{1}\right) \phi\left(x_{2}\right) \psi\left(x_{3}\right) \\
\Psi^{v, a}\left(x_{1}, x_{2}, x_{3}\right)=\phi\left(x_{1}\right) \psi\left(x_{2}\right) \phi\left(x_{3}\right) & \Psi^{d, a}\left(x_{1}, x_{2}, x_{3}\right)=\phi\left(x_{1}\right) \psi\left(x_{2}\right) \psi\left(x_{3}\right) \\
\Psi^{s, d}\left(x_{1}, x_{2}, x_{3}\right)=\psi\left(x_{1}\right) \phi\left(x_{2}\right) \phi\left(x_{3}\right) & \Psi^{h, d}\left(x_{1}, x_{2}, x_{3}\right)=\psi\left(x_{1}\right) \phi\left(x_{2}\right) \psi\left(x_{3}\right) \\
\Psi^{v, d}\left(x_{1}, x_{2}, x_{3}\right)=\psi\left(x_{1}\right) \psi\left(x_{2}\right) \phi\left(x_{3}\right) & \Psi^{d, d}\left(x_{1}, x_{2}, x_{3}\right)=\psi\left(x_{1}\right) \psi\left(x_{2}\right) \psi\left(x_{3}\right),
\end{array}
$$

where all dimensions are dilated similarly and the sampling is done separately along each dimension of the image. The original multispectral image of size $N \cdot N \cdot M$ is filtered into octants of size $\frac{N}{2} \cdot \frac{N}{2} \cdot \frac{M}{2}$. The number of levels is defined similarly to the two-dimensional wavelet transform as illustrated in Fig. 1. 


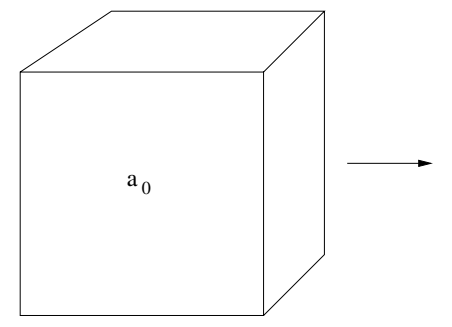

original image

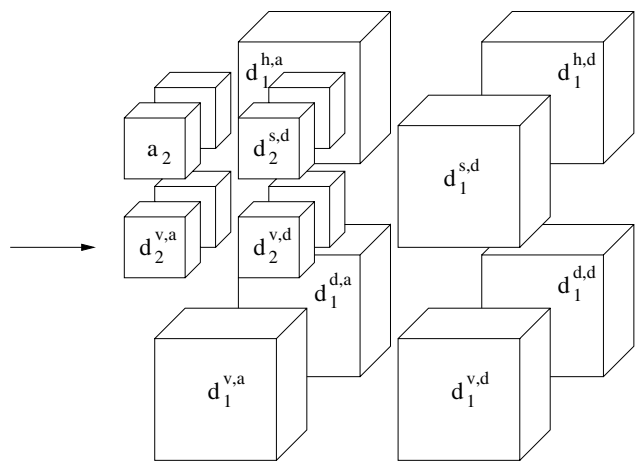

2-level transform

Fig. 1. Three-dimensional wavelet transform applied in two levels. The coefficients $a$ come from the low-pass filtering and the coefficients $d$ come from the high-pass filtering.

\section{Watermarking Techniques}

With RGB-color image watermarking the watermark is normally a binary image. With spectral images, several possibilities exist for the watermark embedding. The watermark itself can be a binary, gray-scale, RGB-color image or even a spectral image with several gray-levels in each band.

\subsection{Embedding a Watermark}

In this study one-band gray-scale image is used as a watermark. The embedding procedure is as follows:

1) Compute a two-dimensional wavelet transform $W_{w t}$ of the watermark $W$. We use only one level. The dimensions of the watermark are $\left(n_{w x}, n_{w y}\right)$.

2) Compute a three-dimensional wavelet transform $I_{w t}$ of the spectral image $I$. The number of levels is matched with the size of the watermark. At the highest level the spatial size of the transformed block is equal to the size of the watermark. Thus the size of the transformed block is $\left(n_{i x}, n_{i y}, n_{i z}\right)$ with $n_{i x}=n_{w x}, n_{i y}=n_{w y}$.

3) Add the transformed values of the watermark $W_{w t}$ to the values of the transformed block $B_{w t}$ resulting to the watermarked block $B_{w t, w m}$,

$$
B_{w t, w m}=B_{w t}+a_{1} \cdot a_{2} \cdot W_{w t}
$$

For each pixel of the watermark a suitable band from the transformed block $B_{w t}$ is selected. First the respective pixels from all the bands inside the transformed block are selected; then the median of the pixels is calculated; finally, the band holding this median is selected. The multiplier $a_{1}$ is defined based on the contrast sensitivity function of the selected band to minimize perceptual error in the embedding procedure [7]. The multiplier $a_{2}$ controls the strength of the watermark. 
4) Reconstruct the spectral image $I_{w m}^{r}$ containing the watermark from the transformed image with the watermarked block $B_{w t, w m}$ by the inverse three-dimensional wavelet transform.

Naturally the reconstructed image $I_{w m}^{r}$ differs from the original image $I$. The difference depends on the strength $a_{2}$ in the embedding of the watermark according to Eq. 4 . The larger the multiplier $a_{2}$ the better the watermark survives from the attacks. If the multiplier $a_{2}$ is small then the watermark is more invisible but at the same time, more vulnerable in attacks. In this study, the multiplier $a_{2}$ as the strength was experimentally defined as $a_{2}=5 \% \cdot \hat{I}$, where $\hat{I}$ is the mean of the original image $I$. Similar rules of thumb are used also in other studies, e.g. see [7]. There is no exact and correct value for $a_{2}$. It should be defined as an optimum of multiple target functions like visibility in the watermarked image, vulnerability in attacks, reproducibility of the watermark and so on.

The multiplier $a_{1}$ depends on the frequency content of the wavelet transformed band $b$. Now $a_{1}$ is defined as

$$
S_{b}=\sum_{\forall u, v} C(u, v)\left|F_{b}(u, v)\right|^{2}, \quad a_{1}=\frac{S_{b}}{\max _{\forall \mathrm{b}} \sqrt{S_{b}}},
$$

where $C(u, v)=5.05 e^{-0.178(u+v)}\left(e^{0.1(u+v)}-1\right)$ is the contrast sensitivity matrix with frequencies $u$ and $v . F_{b}(u, v)$ is the discrete Fourier transform of the band $b$ in the block.

\subsection{Extracting the Watermark}

Extraction of the watermark is an inverse operation to the embedding operation. Thus, the original spectral image is required. Now the extraction equation is

$$
W^{r}=I_{w m}^{r}-I
$$

Since the wavelet transform carries the perfect reconstruction property [12], the difference $W^{r}$ in Eq. 6 contains the information of the embedded watermark. In embedding, the three-dimensional wavelet transform spreads the watermark over the whole image and the extraction procedure collects these spread parts into one original watermark.

\section{Attacks}

Remote sensing is a typical application area for spectral images. Applications may require information only from some bands, so the watermarking procedure should be as robust as possible against cropping. The other typical application for spectral images is classification and thus, the watermarking should not generate e.g. artificial peaks into the spectral domain of the image. Other typical attacks include lossy compression with transform methods; in this study, the principal component analysis and the wavelet transform are used.

After the attack the watermark is extracted from the image and then the quality of the extracted watermark is calculated using the signal-to-noise ratio (SNR). SNR is defined as

$$
S N R=10 \log _{10} \frac{E^{o}}{E^{d}},
$$


where $E^{o}$ is the energy of the original watermark and $E^{d}$ is the energy of the difference between the original watermark and the extracted watermark.

The correlation coefficient also acts as a quality measure between the original watermark and the extracted watermark [7]. The correlation coefficient $c c$ is defined as

$$
c c=\frac{\sum\left(W^{o}-\bar{W}^{o}\right)\left(W^{e}-\bar{W}^{e}\right)}{\sqrt{\sum\left(W^{o}-\bar{W}^{o}\right)^{2}} \sqrt{\sum\left(W^{e}-\bar{W}^{e}\right)^{2}}},
$$

where $W^{o}$ is the original watermark and $W^{e}$ is the extracted watermark. $\bar{W}^{o}$ and $\bar{W}^{e}$ are the respective means.

\section{Experiments}

The experiments had two purposes. Both the embedding/extraction procedure, and then the robustness of the watermark in the image compression were experimented.

Initially, the watermark contained 8 gray-levels, it is shown in Fig. 2 a). The size of the watermark was $n_{w x} \cdot n_{w y}=128 \cdot 128$. The test spectral image was of size $N \cdot N \cdot M=256 \cdot 256 \cdot 32$. The image was extracted from the AVIRIS database, it is called JASPER RIDGE. Band 11 of the image is shown in Fig. 2 b). In Fig. 2 c), we illustrate band 11 of the watermarked image and in Fig. $2 \mathrm{~d}$ ), the average spectrum from the original image and from the watermarked image are shown.

The original image was transformed in two levels with the three-dimensional wavelet transform and the watermark was transformed in one level. Now the spatial size of the approximative block of the spectral image was the same as the spatial size of the approximative block of the watermark. The wavelet was Daubechies orthogonal filter with 4 taps. After embedding the difference between the original image and the reconstructed watermarked image was $S N R=34.16 d B$, see Eq. 7. The energies were calculated from the images and thus, $E^{o}$ corresponded to the energy of the original image and $E^{d}$ corresponded to the energy of the difference between the original image and the reconstructed image.

Image compression was used as a test to evaluate the robustness of the watermark. The watermarked image was compressed with the principal component analysis (PCA) in the spectral domain. Then each eigenimage was compressed with the twodimensional wavelet transform. The number of principal components varied from 4 to 10 and the bit-rate in the wavelet transform was from 0.1 to 8 . The original data had the resolution of 16 bits/sample. The results from this experiment are in Fig. 3 a). The compression ratio is on the horizontal axis and the value of the correlation coefficient $c c$, see Eq. 8, is on the vertical axis. Fig. $3 \mathrm{~b}$ ) shows the extracted watermark after the compression and reconstruction with the compression ratio 16.46. In Fig. $3 \mathrm{c}$ ) the band 11 from the compressed and reconstructed image is shown.

The strength in embedding was varied and the quality of the reconstruction was measured. Also compression experiments with watermarks with different number of gray-levels were performed. The compression ratio for both experiment was 16.46. The results from these experiments are collected into Tables 1 and 2.

In Tables 1 and 2 the compression/reconstruction quality higher than $S N R=30 \mathrm{~dB}$ means negligible visual degradation of the image. The value of the correlation coefficient $c c<0.5$ means clear visual degradation, but still the watermark can be identified. 


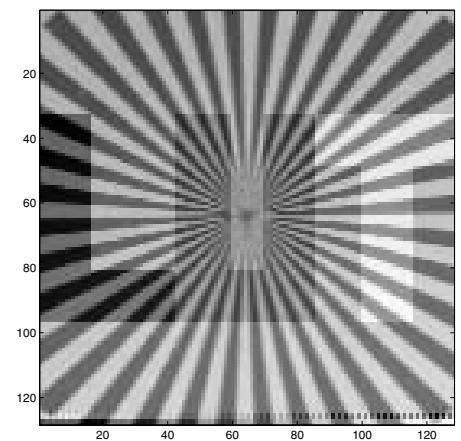

a)

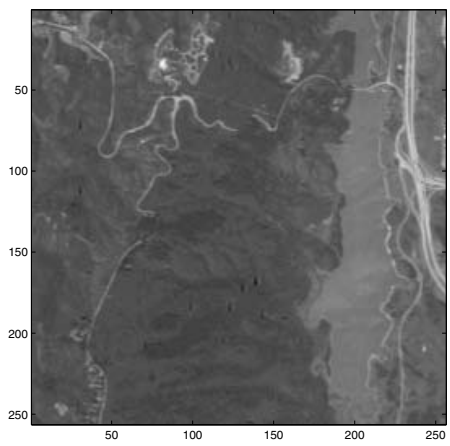

c)

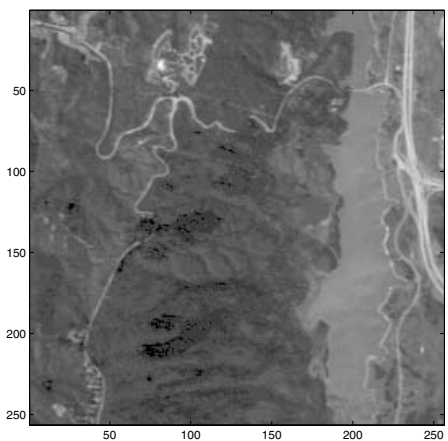

b)

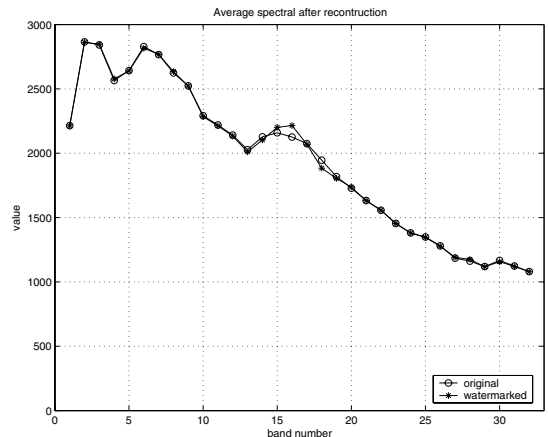

d)

Fig. 2. a) Original watermark with 8 gray-levels. b) Band 11 of the original image. c) Band 11 of the watermarked image. d) Average spectrum from the original image and the watermarked image.

\section{Conclusions}

Watermarking techniques constitute a new area in controlling authorized exploitation of spectral images. We defined the watermarking technique where the watermark is embedded in the three-dimensional wavelet domain of the spectral image. The robustness of the watermark was tested against PCA/wavelet-based compression.

The experiments indicate that the embedding procedure has reasonable behavior concerning the strength in embedding the watermark, see Table 1. Low multipliers in the strength, i.e. values $0.5,1$, and $2 \%$, result in too deficient robustness in compression. The procedure allows watermarks with different number of gray-levels to be embedded in equal quality, only binary watermarks yield too low quality in embedding and compression, see Table 2.

Further research is still needed even though the average results are good. In the experiments we noticed, that some bands carried most of the information of the watermark. For example, band 13 contained $26 \%$ of the watermark and bands from 11 to 16 contained $91.4 \%$ of the watermark. Band 11 contained $10.3 \%$ of watermark. Thus, the embedding should spread the watermark more equally over all bands. This will also 


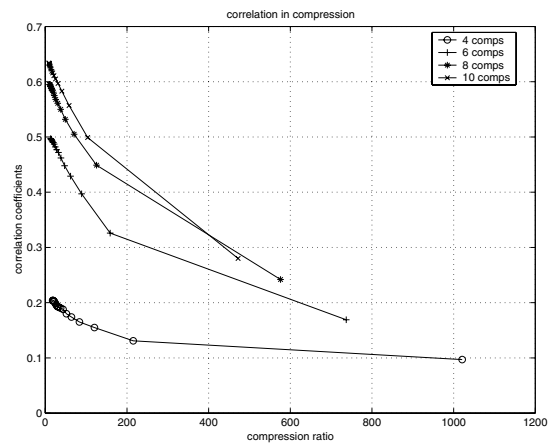

a)

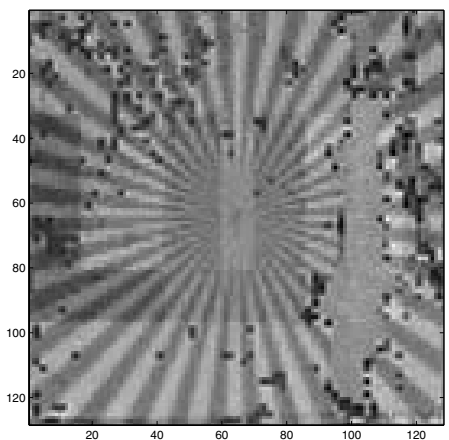

b)

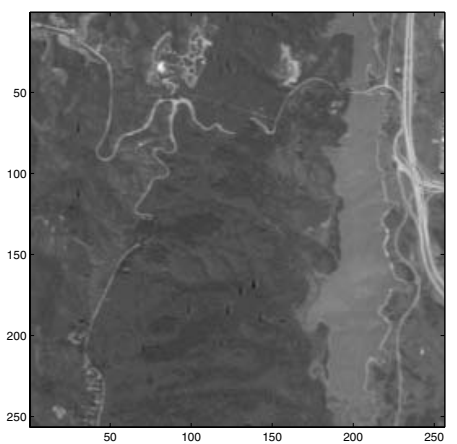

c)

Fig. 3. a) Correlation between the original watermark and the extracted watermark after compression and reconstruction. b) The extracted watermark from the compressed and reconstructed image, $c c=0.613$. c) Band 11 from the compressed and reconstructed image.

enhance the robustness of the watermark against cropping. For spectral images, e.g. remote sensing images, cropping composes a serious problem from the watermarking point of view. Even one band from the spectral image can be cropped or only a small spatial part is of interest. Thus, cropping as an attack will require most thoroughgoing consideration.

\section{References}

1. Artz, D., Digital Steganography: Hiding data within data, IEEE Internet Computing, May/June 2001, pp. 75-80.

2. Podilchuk, C.I., Delp, E.J., Digital watermarking: algorithms and applications, IEEE Signal Processing Magazine, July 2001, pp. 33-46.

3. Barni, M., Bartolini, F., Cappellini, V., Magli, E., Olmo, G., Near-lossless digital watermarking for copyright protection of remote sensing images, Proceedings of IGARSS'02, 2002, pp. 1447-1449.

4. Wolfgang, R.B., Podilchuk, C.I., Delp, E.J., Perceptual watermarks for digital images and video, Proceedings of the IEEE, vol. 87, issue 7, July 1999, pp. 1108-1126. 
Table 1. Modifying the strength in embedding. The number of gray-levels in the watermark was 8 . Columns from left to right: multiplier in $a_{2}$ (see text), quality of embedding, quality of the image after compression, correlation coefficient between the original watermark and the extracted watermark, quality of the extracted watermark.

$\begin{array}{lllll}\begin{array}{l}\text { multiplier embedding compression correlation } \\ \%\end{array} & \text { SNR dB } & \text { SNR dB } & \text { extraction } \\ \begin{array}{lllll}\text { coefficient } & \text { cc } & \text { SNR dB } \\ \hline 0.5 & 54.14 & 43.65 & 0.175 & 25.25 \\ 1 & 48.13 & 43.22 & 0.256 & 26.21 \\ 2 & 42.11 & 42.08 & 0.426 & 27.46 \\ 5 & 34.16 & 33.87 & 0.587 & 28.81 \\ 10 & 28.13 & 34.17 & 0.602 & 29.04 \\ 20 & 22.11 & 28.76 & 0.606 & 29.11 \\ \hline\end{array}\end{array}$

Table 2. Modifying the number of gray-levels of the watermark, multiplier in strength $a_{2}$ is $5 \%$. Columns from left to right: number of gray-levels, quality of embedding, quality of the image after compression, correlation coefficient between the original watermark and the extracted watermark, quality of the extracted watermark.

\begin{tabular}{|c|c|c|c|c|}
\hline gray-levels & SNR dB & SNR dB & coefficient $c c$ & SNR dB \\
\hline 2 & 30.98 & 36.80 & 0.316 & 23.19 \\
\hline 4 & 33.34 & 37.64 & 0.560 & 26.98 \\
\hline 8 & 34.16 & 33.87 & 0.587 & 28.81 \\
\hline 16 & 34.21 & 38.80 & 0.596 & 29.43 \\
\hline 32 & 34.13 & 38.84 & 0.592 & 29.70 \\
\hline 64 & 34.08 & 38.86 & 0.588 & 29.79 \\
\hline
\end{tabular}

5. Cox, I., Miller, M., Bloom, J., Digital Watermarking, Morgan-Kaufmann, San Francisco, USA, 2001.

6. Hsieh, M.-S., Tseng, D.-G., Huang, Y.-H, Hiding digital watermarks using multiresolution wavelet transform, IEEE Transactions on Industrial Electronics, vol. 48, no. 5, Oct. 2001, pp. 875-882.

7. Kundur, D., Hatzinakos, D., A Robust Digital Image Watermarking Method using WaveletBased Fusion, Proceedings of the IEEE Intl. Conference on Image processing, Santa Barbara, California, vol. 1., 1997, pp. 544-547.

8. Cheung, W.N., Digital image watermarking in spatial and transform domains, TENCON Proceedings, vol. 3, 2000, pp. III-374-III-378.

9. Parisis, A., Carre, P., Fernandez-Maloigne, C., Colour watermarking: study of different representation spaces, Proceedings of CGIV, 2002, pp. 390-393.

10. Barni, M., Podilchuk, C.I., Bartolini, F., Delp, E.J., Watermark embedding: hiding signal within cover image, IEEE Comm. Magazine, Aug. 2001, pp.102-108.

11. Ho, A.T.S., Shen, J., Tan, S.H., Kot, A.C., Digital image-in-image watermarking for copyright protection of satellite images using the fast Hadamard Transform, Proceedings of IGARSS'02, 2002, pp. 3311-3313.

12. Daubechies, I., Ten lectures on wavelets, CBMS-NSF, 1992.

13. Mallat, S., A theory for multiresolution signal decomposition: the wavelet presentation, IEEE Transactions on Pattern Analysis and Machine Intelligence, vol. 11, no. 7, July 1989, pp. $674-693$. 